\title{
»Religionspolitik« in Deutschland im europäischen Kontext
}

\section{Die Dynamisierung des Verbältnisses von Staat und Religionen in Deutschland}

»Wir werden den Dialog mit den christlichen Kirchen, Religionsgemeinschaften und religiösen Vereinigungen sowie den freien Weltanschauungsgemeinschaften intensiv pflegen. Sie bereichern das gesellschaftliche Leben und vermitteln Werte, die zum $\mathrm{Zu}$ sammenhalt unserer Gesellschaft beitragen. Wir bekennen uns zum Respekt vor jeder Glaubensüberzeugung. Auf der Basis der christlichen Prägung unseres Landes setzen wir uns für ein gleichberechtigtes gesellschaftliches Miteinander in Vielfalt ein. ${ }^{1}$ Mit dieser Absichtserklärung wird im Koalitionsvertrag von CDU, CSU und SPD vom Herbst 2013 der Abschnitt »Kirchen und Religionsgemeinschaften « eröffnet. Die darin zum Ausdruck gebrachte Wertschätzung für die Religionsgemeinschaften und insbesondere die Kirchen deckt sich - unbeschadet aller Säkularisierungstendenzen und religionskritischen Stimmen in der Öffentlichkeit insbesondere zu »dem Islam« - mit der nach wie vor weit verbreiteten positiven Grundhaltung zu Religion und Kirchen in der deutschen Bevölkerung. Neu ist aber, dass diese Koalitionsvereinbarung erstmals einen eigenständigen längeren Abschnitt zu den Kirchen, Religions- und Weltanschauungsgemeinschaften enthält. Neu ist auch, dass die Koalitionäre darin offensichtlich bemüht sind, der gewachsenen religiösen Vielfalt der deutschen Gesellschaft gerecht zu werden. Einzeln angeführt und damit gewürdigt werden »die christlichen Kirchen und ihre Wohlfahrtsverbände «, die »jüdischen Gemeinden und die jüdische Wohlfahrtspflege«, die »muslimische[n] Vereine und Verbände" sowie - wenn auch nicht ganz so prominent - die »freien Weltanschauungsgemeinschaften.$^{2}$ Vor allem aber fällt auf, dass die Koalitionsvereinbarung inhaltliche Aussagen und Festlegungen zum zukünftigen Verhältnis von Staat und Kirchen bzw. Religionsgemeinschaften macht, die man in den vorhergehenden Koalitionsverträgen so bislang nicht angetroffen hat. Ausdrücklich wird festgehalten: »Das bewährte Staatskirchenrecht in unserem Land ist eine geeignete Grundlage für eine partnerschaftliche Zusammenarbeit mit den Religionsgemeinschaften. $\ll^{3}$ Den Kirchen wird zugesagt, die Koalition halte »am System der Kirchensteuern fest« und werde »die kirchlichen Dienste weiter unterstützen. $\aleph^{4}$ Ebenfalls weitergeführt werden soll die Un-

1 Deutschlands Zukunft gestalten. Koalitionsvertrag zwischen CDU, CSU und SPD. 18. Legislaturperiode, o.O. o.J. (Berlin 2013), S. 113 (PDF), <http://www.bundesregierung.de/Content/ $\mathrm{DE} /$ StatischeSeiten/Breg/koalitionsvertrag-inhaltsverzeichnis.html> am 30.1.2014.

2 Ebd.

3 Ebd.

4 Ebd. 
terstützung für die jüdischen Gemeinden. Und den muslimischen Vereinen und Verbänden wird die Fortsetzung der Deutschen Islamkonferenz in Aussicht gestellt.

Diese Bekräftigungen versprechen eine Fortschreibung des Status quo der Kirchen und stellen die Fortsetzung des eingeschlagenen Weges in Aussicht, weiteren Religionsgemeinschaften und insbesondere dem Islam in seinen verschiedenen Richtungen und Gruppierungen Formen der Kooperation anzubieten. Es könnte nun eingewandt werden, dies sei eigentlich nicht neu, denn für den Kenner der Sachlage enthält der Koalitionsvertrag nur Bekanntes und bereits Praktiziertes. Das Novum liegt freilich in der Tatsache, dass es diesen Abschnitt und seine Aussagen gibt. Es darf nämlich nicht übersehen werden, dass gerade mit den bestätigenden Aussagen zum Status der Kirchen implizit die Botschaft mitgeteilt wird, dass es prinzipiell in der Möglichkeit der Politik liegt, das Verhältnis von Staat und Religionsgemeinschaften zu gestalten - und das heißt, es gegebenenfalls neu und ganz anders als bislang zu bestimmen. Der Religionsabschnitt des Koalitionsvertrags wäre ohne diese unausgesprochene, aber eben doch mitgedachte Unterstellung eines grundsätzlichen politischen Gestaltungsauftrags des deutschen Staates gegenüber den verschiedenen Formen organisierter Religion in der Gesellschaft nicht verständlich. Wer am System der Kirchensteuer öffentlich festhält, signalisiert bzw. unterstellt im gleichen Atemzug, dass es prinzipiell möglich wäre, es zu ändern oder gar abzuschaffen. ${ }^{5}$

Der vorgestellte Abschnitt des jüngsten Koalitionsvertrags und die darin zum Ausdruck kommende Verschiebung hin zu einem aktiveren Politikverständnis gegenüber den Religionsgemeinschaften in Deutschland sind kein zufälliges Politikergebnis. Die Aussagen liegen vielmehr auf der Linie der öffentlichen Debatten sowie gerichtlichen und politischen Entscheidungen der zurückliegenden Jahre. Sie sind ein Ergebnis der nicht zu übersehenden Dynamisierung des Verhältnisses von Politik und Religion in Deutschland, sowohl auf der Ebene des Bundes wie auf der der Bundesländer. Die Kontroversen und Konflikte um die öffentlichen Ausdrucksformen muslimischen Glaubens, etwa um das Tragen des muslimischen Schleiers oder um Moscheebauten, die Kritik an der Art der Finanzierung der beiden großen Kirchen und insbesondere an der Zahlung von Staatsleistungen an Bistümer und Landeskirchen sowie Streitigkeiten um den besonderen Status der Kirchen als Arbeitgeber sind Teil eines generell in Bewegung geratenen Zusammenhangs von Staat und Kirchen bzw. Religionsgemeinschaften. ${ }^{6}$ Diese Entwicklung teilt Deutschland mit einer ganzen Reihe von westlichen Demokratien wie Frankreich, Belgien, den Niederlanden oder der Schweiz, in denen ebenfalls über den

5 Selbstverständlich soll an dieser Stelle keinerlei Aussage seitens des Autors zu den verfassungsund vertragsrechtlichen Rahmenbedingungen und den damit einhergehenden Garantien der Kirchensteuer in Deutschland getroffen werden. Vielmehr geht es darum, die mittlerweile weitreichende Veränderung in der politischen Kommunikation zum Themenkomplex in dieser Einleitung des Hefts sichtbar zu machen.

6 Vgl. die Beiträge in Gert Pickel / Oliver Hidalgo, (Hg.), Religion und Politik im vereinigten Deutschland. Was bleibt von der Rückkebr des Religiösen? (= Politik und Religion), Wiesbaden 2013. 
zukünftigen Weg des politischen und rechtlichen Umgangs mit Kirchen und Religionsgemeinschaften diskutiert und gestritten wird?

In Deutschland ist das Verhältnis von Staat und Kirchen bzw. Religionsgemeinschaften noch bis in die 1990er Jahre hinein sehr stabil gewesen. Es stand selbst im epochalen Umbruch der deutschen Einheit nicht ernsthaft zur Diskussion. Vielmehr erwiesen sich die im Rahmen des Grundgesetzes 1949 geschaffenen Regelungen, die im Anschluss an die Bestimmungen der Weimarer Reichsverfassung maßgeblich zur Befriedung älterer religiös-weltanschaulicher Konflikte im konfessionell geprägten Deutschland beigetragen haben, auch für die vergrößerte Bundesrepublik Deutschland als tragfähige Grundlagen. ${ }^{8}$ Das Modell der grundrechtlich verankerten Religions-, Bekenntnis- und Gewissensfreiheit in Verbindung mit einer kooperativen Trennung von Staat und Kirchen diente auch im Demokratiegründungsprozess der damals neuen Bundesländer als Richtschnur. ${ }^{9}$

Für die jüngst zu beobachtende Dynamisierung des Verhältnisses von Staat und Religionsgemeinschaften kommen zahlreiche Ursachen in Betracht. Zu nennen sind zunächst gesellschaftliche Veränderungsprozesse im religiösen und kulturellen Bereich. Die religiöse Landschaft der Gegenwart ist deutlich vielschichtiger geworden. ${ }^{10}$ Der Trend der anhaltenden Entkirchlichung und einer sich ausbreitenden religiösen Indifferenz einerseits und die Zunahme religiöser, insbesondere muslimischer Minderheiten und deren

7 Veit Bader, Secularism or Democracy? Associational Governance of Religious Diversity (= IMISCOE Research), Amsterdam 2007; Silvio Ferrari / Sabrina Pastorelli, (Hg.), Religion in Public Spaces: A European Perspective (= Cultural Diversity and Law in Association with RELIGARE), Farnham u.a. 2012; Karlies Abmeier / Michael Borchard / Matthias Riemenschneider (Hg.), Religion im öffentlichen Raum (= Religion - Staat - Gesellschaft, Bd.1), Paderborn 2013.

8 Der nach der deutschen Einheit bis Mitte der 1990er Jahre entstandene Rechtsstand ist zusammengefasst in Joseph Listl / Dietrich Pirson, (Hg.), Handbuch des Staatskirchenrechts der Bundesrepublik Deutschland, 2., grundlegend neubearb. Aufl., Berlin 1994-1995. - Die Ausnahme von dieser Regel war die Kontroverse im Land Brandenburg um die Einführung des neuen ordentlichen Unterrichtsfaches »Lebensgestaltung-Ethik-Religionskunde« (LER) an Stelle des Religionsunterrichts, die sich freilich erst 1996 mit dem Streit um die Geltung der Bremer Klausel (Art. 141 GG) zu einem Konflikt von grundsätzlicher staats- und religionspolitischer Bedeutung zuspitzte.

9 Vgl. etwa Joseph Listl, »Das Staatskirchenrecht in den neuen Ländern der Bundesrepublik Deutschland. Die Entwicklung von 1989 bis 1994« in: Essener Gespräche zum Thema Staat und Kirche 29 (1995), S. 160-191; Claudio Fuchs, Das Staatskirchenrecht der neuen Bundesländer (= Jus ecclesiasticum, Bd.61), Tübingen 1999.

10 Vgl. mit weiterer Literatur Gert Pickel / Kornelia Sammet, (Hg.), Religion und Religiosität im vereinigten Deutschland. Zwanzig Jabre nach dem Umbruch (= Veröffentlichungen der Sektion Religionssoziologie der Deutschen Gesellschaft für Soziologie), Wiesbaden 2011; Gert Pickel / Kornelia Sammet, (Hg.), Transformations of Religiosity. Religion and Religiosity in Eastern Europe 1989-2010 (= Veröffentlichungen der Sektion Religionssoziologie der Deutschen Gesellschaft für Soziologie), Wiesbaden 2012; Christof Wolf / Matthias Koenig, (Hg.), Religion und Gesellschaft (= Kölner Zeitschrift für Soziologie und Sozialpsychologie, Sonderheft 53), Wiesbaden 2013; Detlef Pollack / Olaf Müller / Gert Pickel, (Hg.), The Social Significance of Religion in an Enlarged Europe. Secularization, Individualization and Pluralization, Aldershot 2012. 
Interesse an öffentlicher Anerkennung und Teilhabe andererseits haben die älteren politischen und staatskirchenrechtlichen Selbstverständlichkeiten fraglich werden lassen. ${ }^{11}$ Mit dem Rückgang der Kirchlichkeit und der Abnahme der Zahl der Mitglieder in beiden großen Kirchen scheint zudem eine veränderte Positionsbestimmung der Kirchen im Gefüge der gesellschaftlichen Öffentlichkeit einherzugehen. Immer weniger werden die Kirchen als mehr oder weniger autoritative Institutionen sui generis der Verkündigung und Glaubensweitergabe betrachtet, immer stärker dagegen als nach wie vor wichtige zivilgesellschaftliche Akteure unter anderen. ${ }^{12}$ Zugleich werden die damit verbundenen positiven Erwartungshaltungen demokratischer Einstellungs- und Verhaltensweisen auch an die Kirchen und an alle anderen Vergemeinschaftungsformen von Religion herangetragen. ${ }^{13}$ Religion muss heute zivilgesellschaftskompatibel sein, um gesellschaftliche Geltung erlangen zu können.

Im politischen Bereich dürften zunächst die Ausweitung des internationalen Menschenrechtsschutzes und die europäische Integration - und hier insbesondere die nach 1992 im Zuge der Vertiefung der Europäischen Union vergleichsweise rasch entstandenen religionsrechtlichen Regelungen der EU - sowie die indirekten Wirkungen der EUArbeitsmarkt- und Antidiskriminierungspolitik auf das Religionsrecht der Bundesrepublik Deutschland zurückwirken. ${ }^{14}$ Insbesondere kirchliche Dienstleister und Arbeitge-

11 Vgl. Joachim Wieland u.a., Arbeitsmarkt und staatliche Lenkung. Staat und Religion. Berichte und Diskussionen auf der Tagung der Vereinigung der Deutschen Staatsrechtslehrer in Heidelberg vom 6. bis 9. Oktober 1999 (= Veröffentlichungen der Vereinigung der Deutschen Staatsrechtslehrer, H.59), Berlin/New York 2000; Christian Walter, Religionsverfassungsrecht: in vergleichender und internationaler Perspektive (= Ius publicum, Bd.150), Tübingen 2006; Hans Michael Heinig / Christian Walter, (Hg.), Staatskirchenrecht oder Religionsverfassungsrecht? Ein begriffspolitischer Grundsatzstreit, Tübingen 2006; Michael Droege, »Der Religionsbegriff im deutschen Religionsverfassungsrecht - oder: Vom Spiel mit einer großen Unbekannten « in: Mathias Hildebrandt / Manfred Brocker, (Hg.), Der Begriff der Religion (= Politik und Religion), Wiesbaden 2006, S. 159-176.

12 Vgl. mit weiterer Literatur Antonius Liedhegener / Ines-Jacqueline Werkner, (Hg.), Religion zwischen Zivilgesellschaft und politischem System. Befunde - Positionen - Perspektiven (= Politik und Religion), Wiesbaden 2011; Richard Traunmüller, Religion und Sozialkapital. Ein doppelter Kulturvergleich, Wiesbaden 2012.

13 Vgl. etwa Hermann-Josef Große Kracht, »Wie religionsfreundlich darf der moderne Verfassungsstaat sein? « in: Karl Gabriel / Hans-Joachim Höhn, (Hg.), Religion beute - öffentlich und politisch. Provokationen, Kontroversen, Perspektiven, Paderborn 2007, S. 123-139.

14 Diese Europäisierung der staatlichen Religionspolitik ist Teil der Mehrebenenproblematik des Regierens in der Europäischen Union und ihren Mitgliedstaaten. Vgl. Hartmut Behr / Mathias Hildebrandt, (Hg.), Politik und Religion in der Europäischen Union. Zwischen nationalen Traditionen und Europäisierung (= Politik und Religion), Wiesbaden 2006; Matthias Koenig, "Europäisierung von Religionspolitik. Zur institutionellen Umwelt der Anerkennungskämpfe muslimischer Migranten« in: Monika Wohlrab-Sahr / Levent Tezcan, (Hg.), Konfliktfeld Islam in Europa (= Soziale Welt, Sonderbd. 17), Baden-Baden 2007, S. 337-368; Friederike Böllmann, Organisation und Legitimation der Interessen von Religionsgemeinschaften in der euroräischen politischen Öffentlichkeit. Eine quantitativ-qualitative Analyse von Europäisierung als Lernprozess in Religionsorganisationen (= Bibliotheca Academica. Soziologie, Bd.8), Würzburg 2010; Lucian N. Leustean / John Madeley, (Hg.), Religion, Politics and Law in the European 
ber werden mit neuen rechtlichen Anforderungen zur Wettbewerbsfreiheit und Antidiskriminierung am Arbeitsplatz konfrontiert. Außerdem haben weltpolitische Ereignisse wie die Anschläge vom 11. September 2001 in den USA und weitere Terrorangriffe in Madrid, London und Mumbai sowie die zahlreichen medialen Zusammenstöße zwischen westlichen und islamischen Öffentlichkeiten - etwa im Karikaturenstreit von 2005 - die Parameter der politischen Debatten um Integration und gesellschaftlichen Zusammenhalt massiv verschoben. Gegenwärtig werden »der Islam « und »die Religion « häufig als eine gesellschaftlich und politisch konfliktive und daher regelungs- und steuerungsbedürftige Größe dargestellt. Mit einer solchen Sicht geraten Religion und Glaubensäußerungen aber notwendigerweise unter einen gesellschaftlichen Rechtfertigungs- und Veränderungsdruck und damit stärker in den Bereich von Parteienwettbewerb und demokratisch legitimierten Entscheidungsverfahren.

Wie auch immer man die genannten Faktoren im Einzelnen gewichten oder ergänzen mag: In der Summe führen sie zu veränderten Wahrnehmungen von Religion im öffentlichen Raum und zu vermehrten Anforderungen an politische Akteure, sich mit Religionen zu beschäftigen und das eigene politische Handeln zu überprüfen und gegebenenfalls neu zu definieren. Der einst ausgesprochen stabile, weitgehend verrechtlichte und dadurch entpolitisierte Ordnungsrahmen des bundesdeutschen Staatskirchenrechts wird in diesem veränderten Umfeld immer öfter als problematisch oder doch zumindest anpassungsbedürftig an die neue Situation eingestuft. Es schlägt die Stunde der Politik: Neben das bestehende (verfassungs-)rechtliche Arrangement tritt die Idee und der Anspruch, politisches und staatliches Handeln müsse den gesellschaftlichen Ort und rechtlichen Status von Religion bzw. Religionsgemeinschaften als eine eigene Gestaltungsaufgabe wahrnehmen und entsprechend handeln.

\section{2. »Religionspolitik« - Überlegungen zu Definition und Wirkung eines zweischneidigen Begriffs}

Der Ausdruck »Religionspolitik « steht wie kein zweiter für die gerade skizzierte Entwicklung. Noch bis vor kurzem war das Wort Religionspolitik in der deutschen Alltagssprache nicht geläufig. Wenn überhaupt, traf man es in fachwissenschaftlichen Abhandlungen an. Mittlerweile aber ist das Wort aus den Diskursen um Religion und Politik in Deutschland nicht mehr wegzudenken. Wer heute einen Kommentar in einer führenden Tageszeitung mit der Überschrift »Religionspolitiker « betitelt, geht davon aus, verstanden zu werden. ${ }^{15}$ Ende der 1990er Jahre taucht das Wort »Religionspolitik « in in-

Union, London u.a. 2010; Lucian N. Leustean, (Hg.), Representing Religion in the European Union. Does God Matter? (= Routledge Studies in Religion and Politics), London 2012; InesJacqueline Werkner / Antonius Liedhegener, (Hg.), Europäische Religionspolitik. Religiöse Identitätsbezüge, rechtliche Regelungen und politische Ausgestaltung (= Politik und Religion), Wiesbaden 2013.

15 D.D. (Daniel Deckers), »Religionspolitiker« in: $F A Z$ vom 3.2.2013, S. 10. 
nenpolitischen Debatten zum ersten Mal auf. ${ }^{16}$ Soweit derzeit bekannt, hat zuerst das Land Baden-Württemberg den Begriff »Religionspolitik « in einer amtlichen Publikation verwendet. In einem Bericht zur Integration von Muslimen im Jahr 2005 wurde dort im Umfeld des umstrittenen Kopftuchverbots betont pointiert festgestellt: »Das Aufgabenfeld der Religionspolitik ist in Deutschland laut Verfassung und Rechtsprechung eindeutig eine Domäne der Länder. «17 Aus dem weiteren Kontext ergab sich, dass die Autoren des Berichts Religionspolitik zudem als ein eigenständiges Politikfeld auffassten eine Zuschreibung, die damals wohl ebenfalls ein Novum war. Mittlerweile ist in der bundesdeutschen Innenpolitik wie gezeigt nicht mehr strittig, ob es Religionspolitik überhaupt geben soll. Diskutiert wird vielmehr, welche Politiken in Bezug auf Religion in der deutschen Gesellschaft die richtigen sind.

Dabei lohnt es sich, über die Frage des »ob « und die Implikationen des Begriffs »Religionspolitik « noch einmal nachzudenken. Denn der Begriff ist keineswegs unproblematisch, wie ein Blick in die politikwissenschaftliche Debatte lehrt. Zunächst ist auch für die Politikwissenschaft festzustellen, dass die Verwendung von Wort und Begriff recht jungen Datums ist. In den gängigen politikwissenschaftlichen Wörterbüchern und Fachlexika sucht man einen Eintrag »Religionspolitik « vergebens. ${ }^{18}$ Die Konjunktur des Begriffs begann mit einer Reihe von jüngeren Publikationen zur Innenpolitik, die das Wort »Religionspolitik « im Titel führten. ${ }^{19}$ Überraschenderweise wurde der Begriff aber in aller Regel dort nicht näher definiert oder eingeführt. In der älteren wissenschaftlichen Literatur begegnet der Begriff dagegen nur im Kontext von vordemokratischen Herr-

16 Vgl. Leonore Siegele-Wenschkewitz / Friedrich Weber / Karin Weintz, (Hg.), Religionspolitik in der Bundesrepublik Deutschland. Konzepte der im Bundestag vertretenen politischen Parteien, der Bundesregierung, der evangelischen und katholischen Kirche (= Arnoldshainer Texte, Bd.111), Frankfurt a.M. 2000; Burkhard Reichert, »Religionspolitik und Zivilreligion « in: Rolf Schieder, (Hg.), Religionspolitik und Zivilreligion (= Interdisziplinäre Studien zu Recht und Staat, Bd.20), Baden-Baden 2001, S. 233-237; Burkhard Reichert, »Religionspolitik und innerer Friede« in: Ricarda Dill / Stephan Reimers / Christoph Thiele, (Hg.), Im Dienste der Sache. Liber amicorum für Joachim Gaertner, Frankfurt a.M. 2003, S. 563-568; Wolfgang Schäuble, »Gehört Religionspolitik zur europäischen `Staatsräson<? - ihr Nutzen und ihre Gefahren « in: epd-Dokumentation (2007), H. 13, S. 3-8.

17 Staatsministerium des Landes Baden-Württemberg, Muslime in Baden-Württemberg. Bericht für den Ministerrat am 15.3.2005, o.O. o.J. (Stuttgart 2005), S. 45.

$18 \mathrm{Zu}$ den herangezogenen Fachlexika vgl. Antonius Liedhegener, Religion in der vergleichenden Politikwissenschaft. Begriffe - Konzepte - Forschungsfelder « in: Hildebrandt / Brocker, (Hg.), Der Begriff der Religion, aaO. (FN 11), S. 180-182.

19 Vgl. Gerhard Besier / Hermann Lübbe, (Hg.), Politische Religion und Religionspolitik. Zwischen Totalitarismus und Bürgerfreibeit (= Schriften des Hannah-Arendt-Instituts für Totalitarismusforschung, Bd. 28), Göttingen 2005; Anselm Doering-Manteuffel / Kurt Nowak (Hg.), Religionspolitik in Deutschland. Von der früben Neuzeit bis zur Gegenwart, Martin Greschat zum 65. Geburtstag, Stuttgart/Berlin/Köln 1999; Rolf Schieder, (Hg.), Religionspolitik und Zivilreligion (= Interdisziplinäre Studien zu Recht und Staat, Bd. 20), Baden-Baden 2001; Leonore Siegele-Wenschkewitz, »Einführung in die Tagung « in: dies. / Weber / Weintz, (Hg.), Religionspolitik in der Bundesrepublik Deutschland, aaO. (FN 16), S. 17-22. 
schaftsformen oder modernen Diktaturen. ${ }^{20}$ Wie fremd der Begriff der bundesdeutschen Tradition der kooperativen Trennung von Staat und Kirchen bis vor Kurzem noch war, beweist ein Beitrag des renommierten Juristen und ehemaligen Bundesverfassungsrichters Ernst Wolfgang Böckenförde aus dem Jahr 2000, in dem er die Frage nach der Notwendigkeit einer neuen Religionspolitik in Deutschland noch folgendermaßen formulierte: »Kann es eine staatliche Religionspolitik überhaupt noch geben, kann Religionspolitik noch Thema für eine politische Partei sein? Ist nicht mit der Anerkennung der Religionsfreiheit im Grundgesetz alles Notwendige zum Verhältnis von Staat und Religion gesagt, so dass eine staatliche Religionspolitik darüber hinaus eine Einmischung des Staates in die Freiheit und Unabhängigkeit der Religionsgemeinschaften bedeutet, womöglich sogar eine Verletzung der Trennung von Staat und Kirche? ${ }^{21}$ Seine Antwort lautete damals, dass unter Umständen eine parteipolitisch formulierte und staatlich verantwortete Religionspolitik dem Grundsatz nach im Rahmen des Grundgesetzes möglich und unter Umständen geboten sein könne. ${ }^{22}$ Allerdings zog er den Kreis möglicher politischer Entscheidungen sehr eng. Gewisse Handlungsspielräume attestierte er der Politik in religionsbezogenen Fragen immer dann, wenn religiöse oder religiös motivierte Aktivitäten sich mit der säkularen Rechtsordnung des Staates überschneiden, d.h. vor allem im Sinne der res mixtae von Staat und Kirchen im Sozial-, Gesundheits-, Schulund Kulturbereich sowie bei der Anstaltsseelsorge. Vor dem Hintergrund der verfassungsrechtlichen Grundlage des Menschen- und Grundrechts der Religionsfreiheit und der kooperative Trennung (Art. 4 GG und Art. 140 GG in Verbindung mit den entsprechenden Artikeln der Weimarer Reichsverfassung sowie Art. 3 GG und Art. 33 GG) sah Böckenförde solche Spielräume allerdings als gering an. ${ }^{23}$

Andere gingen weiter. Claus Leggewie konstatierte 2003, dass sich die »Reibungen im Verhältnis zwischen Muslimen bzw. muslimischen Gemeinschaften und der deutschen Rechtsordnung [...] mittlerweile akkumuliert « ${ }^{24}$ hätten. Leggewie, der sich intensiv mit der rechtlichen und politischen Lage des Islams in Deutschland beschäftigt hatte ${ }^{25}$, trat nachdrücklich für eine »Neuregulierung des Verhältnisses von Religion und Politik so-

20 Hans Jürg Braun / Erich Bryner / Norbert Meienberger, Religionskritik und Religionspolitik bei Marx, Lenin, Mao, Zürich 1985; Hans Buchheim, Glaubenskrise im Dritten Reich. Drei Kapitel nationalsozialistischer Religionspolitik, Stuttgart 1953; Wilhelm Enßlin, Die Religionspolitik des Kaisers Theodosios des Großen, München 1953; Ingetraut Ludolphy, Die Voraussetzungen der Religionspolitik Karls V., Berlin 1965.

21 Ernst-Wolfgang Böckenförde, »Notwendigkeit und Grenzen staatlicher Religionspolitik « in: Wolfgang Thierse, (Hg.), Religion ist keine Privatsache, Düsseldorf 2000, S. 173-184, hier S. 173.

22 Vgl. ebd., S. 177-179.

23 Die engen Grenzen, die Böckenförde für eine Religionspolitik durch Staat und Parteien sieht, kommen in der äußerst vorsichtigen Gesamtbewertung zum Ausdruck. Vgl. ebd., S. 177.

24 Claus Leggewie, »Kirche oder Sekte? Muslime in Deutschland und den USA « in: Michael Minkenberg / Ulrich Willems (Hg.), Politik und Religion (= Politische Vierteljahresschrift, Sonderheft 33), Wiesbaden 2003, S. 164-183, 176.

25 Claus Leggewie / Angela Joost / Stefan Rech, Der Weg zur Moschee: Eine Handreichung für die Praxis, Bad Homburg 2002; Leggewie, Kirche oder Sekte?, aaO. (FN 24). 
wie Staat und Kirche in Deutschland $\aleph^{26}$ ein. Unter Hinweis auf die religionsförderliche strikte Trennung von Staat und Religion in den USA forderte er eine deutlichere Trennung für Deutschland. Damit solle das »Quasi-Staatskirchensystem der Bundesrepublik «27 überwunden und die prekäre Lage des Islams im bundesdeutschen » Kirchenkartell $\ll^{28}$ verbessert werden. Ähnliche Forderungen wurden auch von anderen Politikwissenschaftlern erhoben. ${ }^{29}$ Vertreter einer grundrechtsorientierten liberalen politischen Theorie argumentierten hingegen, dass gerade eine solche staatliche Religionspolitik auf der Basis politischer Mehrheiten minderheitenfeindliche Politikergebnisse erst ermöglichen würde. ${ }^{30}$

Vor diesem Hintergrund wird einsichtig, dass der Frage nach der jeweiligen Definition des Begriffs »Religionspolitik « in Forschung und Praxis großes Gewicht beizumessen ist. In ihr schlägt sich das jeweilige Politikverständnis und damit immer auch ein gesellschaftspolitischer Gestaltungwille im Zueinander von Politik und Religionsgemeinschaften nieder. In der Politikwissenschaft verbreitet ist mittlerweile jene Definition, die Ulrich Willems vorgeschlagen hat. Er definiert Religionspolitik als all diejenigen »politischen Prozesse und Entscheidungen, in denen die religiöse Praxis von Individuen einschließlich ihrer kollektiven Ausdrucksformen sowie der öffentliche Status, die Stellung und die Funktion von religiösen Symbolen, religiösen Praktiken und Religionsgemeinschaften in politischen Gemeinwesen geregelt werden. ${ }^{31}$ Diese Definition ist in Bezug auf den Phänomenbereich breit angelegt. Sie umfasst alle Formen der individuellen, kollektiven und korporativen Religionsausübung und lässt die Art der politischen Regulierung und die dazu möglichen bzw. legitimen Verfahren und Instrumente offen. Sie eignet sich damit durchaus für empirische und vergleichende Studien zur Religionspolitik. Normativ angewendet eröffnet eine solche Definition aber der politischen Regulierung

26 Ebd., S. 182.

27 Ebd., S. 172.

28 Ebd., S. 174 (im Original als Überschrift hervorgehoben).

29 Vgl. Volker von Prittwitz, "Zivile oder herrschaftliche Religion? Fundamentalismus, Religionsfreiheit und Verantwortung des zivilen Staates « in: APuZ (2002) B18, S. 33-38; Ulrich Willems, »Religionspolitik in der Bundesrepublik Deutschland 1945-1999. Die politische Regulierung der öffentlichen Stellung von Religion und Religionsgemeinschaften « in: ders., (Hg.), Demokratie und Politik in der Bundesrepublik 1949-1999, Opladen 2001, S. 137-160, 158-160; etwas modifiziert Ulrich Willems, »Religion als Privatsache? Eine kritische Auseinandersetzung mit dem liberalen Prinzip einer strikten Trennung von Politik und Religion « in: Minkenberg / Willems, (Hg.), Politik und Religion, aaO. (FN 24), S. 107-108. Mit anderer Stoßrichtung, nämlich der bewussten Förderung zivilgesellschaftsverträglicher Ausformungen von Religion, spricht auch Rolf Schieder von der Notwendigkeit der Religionspolitik. Vgl. Rolf Schieder, Wieviel Religion verträgt Deutschland?, Frankfurt a.M. 2001, bes. S. 26-28.

30 Hauptuntersuchungsgegenstand waren die diversen Verbote des muslimischen Schleiers für Lehrinnen in fast allen westdeutschen Bundesländern und aller religiösen Zeichen im öffentlichen Dienst des Landes Berlin der Jahre 2004 bis 2006. Vgl. Rolf Schieder, »Die Zivilisierung der Religionen « in: APuZ (2007) B6, 17-24; Rolf Schieder, Sind Religionen gefährlich?, Berlin 2008; Antonius Liedhegener, »Religionsfreiheit und die neue Religionspolitik. Mehrheitsentscheide und ihre Grenzen in der bundesdeutschen Demokratie« in: Zeitschrift für Politik 55 (2008), S. 84-107.

31 Willems, Religionspolitik in der Bundesrepublik Deutschland, aaO. (FN 29), S. 137. 
von Religion und damit den politischen Akteuren einen weiten Raum. Im Rahmen von Demokratien zählen dann auch das Recht auf Religionsfreiheit, seine Auslegung und Anwendung zu den »letztendlich politisch zu entscheidenden Fragen « ${ }^{32}$. Auf mittlere Sicht wäre Religionspolitik dann konsequenterweise als ein eigenständiges Politikfeld aufzufassen bzw. zu konstituieren, das sich grundsätzlich kaum oder gar nicht von anderen Policy-Bereichen demokratischer Innenpolitik unterscheidet. ${ }^{33}$

Eine solche Definition relativiert aber - zumindest implizit - die Bedeutung der Grund- und Menschenrechte erheblich, weil sie Grundrechte als wechselseitig zugestandene und daher politisch aushandelbare Rechte der Gesellschaftsmitglieder auffasst und sie damit prinzipiell der freien politischen Entscheidung unterwirft. Eine solche Definition läuft Gefahr, dass die geforderte »neue Religionspolitik « im Zweifelsfall, und d.h. konkret im politischen Konfliktfall, faktisch auf den Primat der Mehrheitsregel hinausläuft. Und in der Tat haben sich in der politischen Praxis auch in der Gegenwart nicht wenige der parlamentarischen Entscheidungen und direktdemokratischen Abstimmungen zu religionspolitischen Themen als freiheits- und minderheitenfeindlich erwiesen. ${ }^{34}$

Daher scheint es geboten, hier vorsichtiger bzw. präziser zu definieren. Ein Vorschlag lautet, einen weiten von einem engen Begriff von Religionspolitik zu unterscheiden. Bezogen auf demokratische Systeme soll der Begriff im weiteren Sinne sowohl alle religionsbzw. religionsverfassungsrechtlichen Bestimmungen als auch alle in diesem Rahmen stehenden (höchst-)richterlichen, (partei-)politischen und administrativen Festlegungen und Maßnahmen zum Umgang mit religiös-weltanschaulicher Vielfalt in freiheitlichen Gesellschaften umfassen. In Abgrenzung zu den geläufigen, ihrerseits je unterschiedlich konnotierten juristischen Begriffen des Staatskirchenrechts, Religionsrechts oder Religionsverfassungsrechts akzentuiert der weite Begriff der Religionspolitik das in einem allgemeinen Sinne politische Handeln der Judikative und der Exekutive und weniger jenes der Legislative. Der Begriff der Religionspolitik im weiteren Sinne macht das gestalterische Element im Rahmen der Freiheitsrechte und verfassungsrechtlichen Ordnung und damit die Akteursqualität der beteiligten Institutionen und ihrer Vertreter sichtbar. Die weite Definition wirft die Frage nach der Ausgestaltung des religiös-weltanschaulichen Miteinanders der Gesellschaft im Lichte der bestehenden Verfassung und geltenden rechtlichen Bestimmungen zum Verhältnis von Staat und Religionsgemeinschaften auf.

32 Willems, Religion als Privatsache, aaO. (FN 29), S. 318.

33 Ulrich Willems / Michael Minkenberg, »Politik und Religion im Übergang - Tendenzen und Forschungsfragen am Beginn des 21. Jahrhunderts « in: Willems / Minkenberg, (Hg.), Politik und Religion, aaO. (FN 24), S. 13-41, hier S. 15-16. Ob es tatsächlich sinnvoll ist, von einem eigenständigen Politikfeld zu sprechen, ist theoretisch wie empirisch eine offene Frage. Vgl. Andreas Pesch, Das islamische Kopftuch als Gegenstand der Religionspolitik in Frankreich. Ein Deutungsstreit, seine Akteure, Bedingungen und Folgen, Heidelberg (Diss. masch.) 2011, S. 18-20.

34 Vgl. Adrian Vatter, (Hg.), Vom Schächt-zum Minarettverbot. Religiöse Minderbeiten in der direkten Demokratie, Zürich 2010, bes. S. 264-290; Antonius Liedhegener, »Von Kopftüchern, Schleiern und Baskenkappen in Europas Demokratien. Zum Verhältnis von >Religionspolitik und >Körperpolitik in der Gegenwart « in: Monika Glavac / Anna-Katharina Höpflinger / Daria Pezzoli-Olgiati, (Hg.), Second Skin. Körper, Kleidung, Religion, Göttingen 2013, S. 253-278. 
In diesem weiten Sinne wird es - ganz im Sinne Böckenfördes - ein gewisses Maß an Religionspolitik in verfassungsstaatlichen Demokratien immer geben. Denn trotz Grundrechtsschutz und Religionsfreiheit bleibt das Problem des »Ausgleichs und $\mathrm{Zu}$ sammenführens weltlich-politischer und geistlich-religiöser Angelegenheiten und Gesichtspunkte « bestehen, so dass »staatliche Regelungen und Maßnahmen in bestimmten Bereichen immer auch ein Stück Religionspolitik enthalten. ${ }^{35}$ Ziele und Gestaltungsspielräume dieser Religionspolitik sind aber durch den vorgängigen Grundrechtsschutz der Religionsfreiheit deutlich enger gezogen, als es in der zuerst angeführten Definition der Fall ist.

Der Begriff der Religionspolitik im engeren Sinne meint all jene politischen Entscheidungsprozesse über religionsrelevante Sachverhalte innerhalb eines gegebenen politischen Systems, die im Kern auf der Anwendung des demokratischen Mehrheitsprinzips beruhen. Religionspolitik im engeren Sinne meint, dass neben oder an die Stelle der religionsrechtlichen Bestimmungen der Verfassung und der geltenden vertragsrechtlichen und einfachrechtlichen Regelungen des Verhältnisses von Staat und Religionen eine spezielle, von Parteien und Parlamenten sowie in direktdemokratischen Abstimmungen formulierte und zu verantwortende Politik tritt. Religionspolitik im engeren Sinne stellt also darauf ab, dass das Verhältnis von Staat, Gesellschaft und Religionsgemeinschaften seitens des politischen Systems durch Parlaments- und Mehrheitsbeschlüsse mehr oder weniger einseitig ausgestaltet werden kann oder - normativ gewendet - auszugestalten sei. Religionspolitik im engeren Sinne ist innenpolitisch in der Regel der brisantere Fall, denn sie greift im Zweifelsfalle einseitig und ohne Beteiligung der Betroffenen in die Handlungsräume und -möglichkeiten von Einzelnen, religiösen Gruppen und Gemeinschaften ein.

\section{Religionspolitik in der deutschen Innenpolitik der Gegenwart - zu diesem Heft}

In der aktuellen Forschung sind Fragen des Verhältnisses von Staat und Religionen wiederholt und vor allem vergleichend angegangen worden. Erhebliche Anstrengungen sind unternommen worden, um im internationalen Vergleich vor allem liberaler Demokratien die historisch gewachsenen, großen Unterschiede zwischen den jeweiligen politischen Systemen durch aussagekräftige Typologien zu systematisieren. ${ }^{36}$ Dadurch soll es einerseits möglich werden, die institutionellen Bedingtheiten der religiösen Verhältnisse in einzelnen Ländern besser zu erfassen, und andererseits den Einfluss von religiösen Traditionen anhand der Institutionenordnung von Staat und Kirche/Religionen auf aktuelle Policies etwa in der Sozial- oder Lebensschutzpolitik nachzuweisen. Im Zuge dieser Forschungen sind die bestehenden religionsrechtlichen Bestimmungen und staatlichen

35 Böckenförde, Notwendigkeit und Grenzen staatlicher Religionspolitik, aaO. (FN 21), S. 177.

36 Vgl. mit weiterer Literatur den Überblick in Richard Traunmüller, »Zur Messung von StaatKirche-Beziehungen: Eine vergleichende Analyse neuerer Indizes « in: Gert Pickel / Susanne Pickel, (Hg.), Indizes in der Vergleichenden Politikwissenschaft (= Zeitschrift für Vergleichende Politikwissenschaft, Sonderheft 2), Wiesbaden 2012, S. 207-232. 
Politiken gegenüber Mehrheits- und Minderheitsreligionen und weltanschaulichen Gruppierungen immer differenzierter erfasst worden. Insbesondere die Arbeiten von Jonathan Fox haben gezeigt, dass die Idee der strikten Trennung von Staat und Religion in der Realität der allermeisten Staaten dieser Erde keine Entsprechung findet. ${ }^{37}$ Seine Daten zur politisch-rechtlichen Regulierung von Religion in über 170 Länder zeigen eindrucksvoll, dass umfangreiche staatliche Regulierungen des religiösen Bereichs weltweit an der Tagesordnung sind. Das gilt auch und gerade für die älteren liberalen Demokratien Europas, deren jeweilige historische Entwicklung des Verhältnisses von Staat und Kirche sich bis heute in unterschiedlichen Staat-Religionen-Arrangements niederschlägt.

Mit diesem unbestreitbaren Fortschritt der komparativen Forschung ist aber auch eine gewisse Schieflage in der politikwissenschaftlichen Auseinandersetzung mit religionspolitischen Fragen eingetreten. Es fehlt derzeit an Versuchen, Religionspolitik im weiteren wie im engeren Sinne speziell für einzelne, konkrete politische Systeme zusammenhängend und umfassend in den Blick zu nehmen und in ihren Bezügen zu anderen Politikfeldern wie der Migrations- und Integrationspolitik und länderspezifischen Akteurskonstellationen und politischen Gelegenheitsstrukturen zu untersuchen. Diese Aufgabe scheint aber dringlich, weil die verschiedenen Staaten Europas ja in der Tat nach wie vor in hohem Maße unterschiedliche religionsrechtliche Arrangements besitzen, gleichzeitig aber - wie eingangs für den Fall der Bundesrepublik Deutschland illustriert - allenthalben zu beobachten ist, dass sich Parteien und Regierungen anschicken, die bestehenden Arrangements durch religionspolitische Entscheidungen an veränderte gesellschaftliche Rahmenbedingungen anzupassen oder gar neue Konstellationen zu schaffen..$^{38}$ Gefragt und nötig sind also sowohl empirisch belastbare komparative Studien zu den jeweiligen Arrangements und religionspolitischen Maßnahmen, als auch und vor

37 Jonathan Fox hat seine jahrelangen Forschungen dazu in einem Überblickswerk zusammengestellt. Vgl. Jonathan Fox, An Introduction to Religion and Politics. Theory and Practice (= Routledge Studies and Politics), Oxon/New York 2013. Die Daten seines Religion and State Project sind im Internet verfügbar unter <http://www.thearda.com/ras/>; vgl. auch Jonathan Fox, $A$ World Survey of Religion and the State, Cambridge 2008.

38 So hat etwa in Frankreich die konservative Regierung von Nicolas Sarkozy die Beziehungen zu den Religionsgemeinschaften zu verbessern und zu gestalten versucht. Sarkozy persönlich setzte sich für das neue Konzept der offenen oder positiven Laïcité ein. Im scharfen Gegensatz dazu will die sozialistische Nachfolgeregierung unter François Hollande die dadurch entstandenen kooperativen Strukturen nun wieder auflösen und zu einer strikten Trennung im Sinne der klassischen Laïcité zurückkehren. Die neuerliche Politisierung einer älteren Konfliktlinie unter veränderten Bedingungen ist unübersehbar. Vgl. Nicolas Sarkozy, Der Staat und die Religionen. Gespräche mit dem Philosophen Thibaud Collin und dem Dominikanerpater Philippe Verdin, aus dem Franz. übersetzt von Gaby Wurster, Hannover 2008; Alois Müller, »Wie laizistisch ist Frankreich wirklich? Von der kämpferischen zur offenen Laizität in: Manfred Brocker / Hartmut Behr / Mathias Hildebrandt, (Hg.), Religion-Staat-Politik. Zur Rolle der Religion in der nationalen und internationalen Politik (= Religion und Politik), Wiesbaden 2003, S. 70-82; Frank Schenker, »Umkämpfte Laïcité. Eine Policy-Analyse zum Verbot auffälliger religiöser Zeichen an Frankreichs öffentlichen Schulen « in: Felix Heidenreich, (Hg.), Staat und Religion in Frankreich und Deutschland - L'Etat et la religion en France et en Allemagne, Berlin 2008, S. 176-199. 
allem fallspezifisch vergleichende Analysen zu den Kontextbedingungen, Akteuren, politischen Prozessen und Policy-Inhalten der Religionspolitik der Gegenwart.

An dieser Stelle setzt das vorliegende Themenheft an. Die folgenden vier Beiträge stellen die Religionspolitik der Bundesrepublik Deutschland und ihre europäischen Kontexte als solche erstmals dezidiert in den Mittelpunkt der Untersuchung. Sie möchten einen Beitrag dazu leisten, die Umrisse und Entwicklungsrichtung der aktuellen Religionspolitik in Deutschland sichtbar zu machen und auf ihre religiösen und zivilgesellschaftlichen Bedingungsfaktoren und europäischen Zusammenhänge aufmerksam zu machen. Gert Pickel (Leipzig) geht der Frage nach, ob und wie die religiöse Pluralisierung in der Bundesrepublik Deutschland einen Wandel der politischen Kultur herbeigeführt hat und welche Rolle dieser Wandel für das Verständnis der aktuellen religionspolitischen Lage in Deutschland spielt. Richard Traunmüller (Essex, GB) stellt - aufbauend auf die Daten von Fox und eigene Erhebungen - die deutsche Religionspolitik in den allgemeinen europäischen Kontext und analysiert mit quantitativen Methoden und Modellen die Entwicklungsrichtung der religionspolitischen Regulierungen in Europa auf mögliche Konvergenzen zwischen den Staaten. Antonius Liedhegener (Luzern, CH) vergleicht im Rahmen eines strukturell-funktionalen Ansatzes von Politik die wichtigsten religionspolitischen Entscheidungen in der Bundesrepublik Deutschland und der Schweiz seit 1990 und überprüft damit verschiedene in der Debatte geläufige Thesen zu Ursprung, Inhalt und Intention aktueller Religionspolitik. Frank Schenker (Jena/Leipzig) präsentiert die Befunde einer umfassenden Recherche zu den innerparteilichen Strömungen und Forderungen nach einem neuen Laizismus und einer strikteren Trennung von Staat und Religion in Deutschland. Auf der Basis von Partei- und Wahlprogrammen sowie zahlreichen Internetquellen beantwortet er die Frage, wie groß der parteipolitische Veränderungsdruck auf das bestehende Arrangement von Staat und Religionsgemeinschaften in Deutschland derzeit und in naher Zukunft ist bzw. sein wird.

Alle Beiträge verdanken ihre Entstehung dem DVPW-Arbeitskreis »Politik und Religion/Politics and Religion «, der sich in seinen Jahrestagungen und Kooperationsverantstaltungen im Rahmen der DVPW-Sektion für Vergleichende Politikwissenschaft mit dem Feld der Religionspolitik wiederholt auseinandergesetzt hat. Die konkrete Anregung zu diesem Heft geht auf Manfred Brocker (Eichstätt) zurück, dem dafür herzlich gedankt sei. Ebenfalls ein herzliches Dankeschön gilt den Beiträgern für die reibungslose und konstruktive Zusammenarbeit und vor allem Frau Laura Lots (Luzern, CH), die neben ihrer laufenden Abschlussarbeit im Joint Degree Master »Religion - Wirtschaft Politik« die Beiträge umsichtig lektoriert und korrigiert hat.

\section{Zusammenfassung}

Bis vor kurzem war das Wort »Religionspolitik « in der deutschen Öffentlichkeit wie in der Politikwissenschaft ungebräuchlich. Ausgehend vom aktuellen Beispiel des im Dezember 2013 geschlossenen Koalitionsvertrags zeigt dieser Beitrag, dass »Religionspolitik« dem Wort und der Sache nach mittlerweile in der politischen Realität in Deutschland 
angekommen ist. Mit zahlreichen anderen europäischen Demokratien teilt Deutschland die Erfahrung, dass das lange Zeit stabile Verhältnis von Staat und Kirche/Religion durch Säkularisierung und Migration unter Druck und in Bewegung geraten ist. Für die Bewertung und Gestaltung dieses Wandels spielt die jeweilige Definition des Begriffs »Religionspolitik « eine erhebliche Rolle. Der Beitrag zeigt daher mögliche Definitionen und ihre Implikationen für die Art und Weise der zukünftigen Ausgestaltung des Verhältnisses von Staat und Religionen auf. Er skizziert knapp den Stand der vergleichenden empirischen Forschung zum Thema und verortet die nachfolgenden vier Beiträge des Themenhefts in diesem Zusammenhang.

\section{Summary}

Until recently, the phrase » Religionspolitik «(religion policy) was not used in the German public as well as in political science. Starting from the current example of the coalition agreement signed in Berlin in December 2013, the article demonstrates that religion policy has meanwhile reached the political reality in Germany. Similar to many other European democracies, secularization and migration brought pressure on the well-established field of state and churches/religion and stimulated significant changes. For any normative assessment or practical governance of this development, the chosen definition of $\gg$ Religionspolitik « plays a crucial role. Thus, the article presents differing definitions and their implications on how to deal with state-religion affairs in future. The article also sketches the state of comparative empirical research on this topic and situates the four articles of the special issue in this context.

Antonius Liedhegener, »Religion Policy« in Germany in European Perspective 